\title{
ANÁLISIS DE LOS STAKEHOLDERS COMO INSUMO DE ENTRADA EN EL DISEÑO DE UN PLAN PUBLICITARIO
}

\author{
AUTORES: Julio Cesar González Bennett ${ }^{1}$ \\ Jackson Vladimir Lino Figueroa ${ }^{2}$ \\ Laura Patricia Muñiz Jaime ${ }^{3}$ \\ María Leonor Parrales Poveda ${ }^{4}$
}

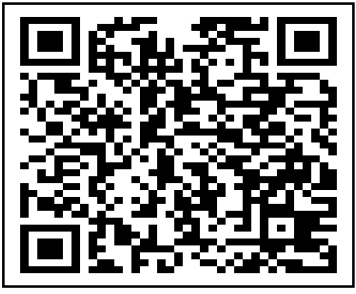

\section{DIRECCIÓN PARA CORRESPONDENCIA: (gonzalez-julio7195@unesum.edu.ec)}

Fecha de recepción: 12/03/2021

Fecha de aceptación: 20/08/2021

\section{RESUMEN}

Los grupos de interés de una empresa pueden ser internos o externos y se les denomina Stakeholders, mismos que no siempre son considerados en la toma de decisiones de las organizaciones. En tal sentido, determinar la importancia de los Stakeholders como insumo de entrada para el diseño del plan publicitario es vital. Por ello, se consideró el caso de varias empresas latinoamericanas y empresas ecuatorianas de diferente razón social, que analizadas desde un diseño de investigación de tipo descriptiva apoyada en la revisión bibliográfica de las posturas que han surgido en relación a los Stakeholders demuestran su incidencia sobre los planes de publicidad. Los resultados permitieron exponer las bases conceptuales que argumentan la propuesta de incluir a este grupo de interés como factor de éxito en el diseño publicitario de las empresas. De donde se concluye que su análisis ayuda a la empresa a definir de manera efectiva sus procesos de ventas encaminados a la satisfacción de las necesidades de las partes interesadas generando ventajas competitivas en el mercado donde esta se desenvuelve.

PALABRAS CLAVE: percepción de valor, toma de decisiones, Stakeholders.

\footnotetext{
${ }^{1}$ Profesional en Formación de la Carrera Administración de Empresas, Facultad Ciencias Económicas-Universidad Estatal del Sur de Manabí, Jipijapa - E-mail: gonzalez-julio7195@unesum.edu.ec

${ }^{2}$ Profesional en Formación de la Carrera Administración de Empresas, Facultad Ciencias Económicas-Universidad Estatal del Sur de Manabí, Jipijapa - E-mail: lino-jackson3300@unesum.edu.ec

3 Economista, Magister en Contabilidad y Auditoría Facultad de Ciencias Administrativas y Económicas, Universidad Estatal del Sur de Manabí, Ecuador - E-mail: lauramuñiz@unesum.edu.ec

${ }^{4}$ Doctora en Ciencias Pedagógicas; Magister en Docencia Universitaria e Investigación Educativa; Magister en Administración de Empresas; Economista. Docente de la Universidad Estatal del Sur de Manabí, Ecuador - E-mail: maria.parrales@unesum.edu.ec
} 
Julio C. González Bennett, Jackson V. Lino Figueroa, Laura Patricia Muñiz Jaime, María Leonor Parrales Poveda

\title{
ANALYSIS OF STAKEHOLDERS AS AN INPUT IN THE DESIGN OF AN ADVERTISING PLAN
}

\begin{abstract}
The interest groups of a company can be internal or external and are called Stakeholders, which are not always considered in the decision-making of organizations. In this sense, determining the importance of Stakeholders as input for the design of the advertising plan is vital. For this reason, the case of several Latin American companies and Ecuadorian companies of different social names was considered, which, analyzed from a descriptive research design supported by the bibliographic review of the positions that have emerged in relation to Stakeholders, demonstrate their impact on the advertising plans. The results allowed to expose the conceptual bases that argue the proposal to include this interest group as a factor of success in the advertising design of companies. From which it is concluded that its analysis helps the company to effectively define its sales processes aimed at satisfying the needs of interested parties, generating competitive advantages in the market where it operates.
\end{abstract}

KEYWORDS: value perception, decision making, Stakeholders.

\section{INTRODUCCIÓN}

El contexto actual del mercado se muestra cada vez más competitivo y obliga a las organizaciones a estudiar profundamente su actuación en el escenario frente a los compradores y demás vendedores, a fin de plantear estrategias que le permitan ir hacia adelante en un ambiente dinámico y cambiante, en este sentido el presente estudio toma por objeto la importancia de la gestión de los Stakeholders en 5 empresas latinoamericanas y 5 empresas ecuatorianas con diferentes giros de negocio y diferentes grupos de interés.

Considerando que los grupos de interés, analizados desde perspectiva organizacional se los describe como Stakeholders, que a criterio de, Moreno, R. (2015) "las empresas desarrollan operaciones deben atender sus tendencias para evitar riesgos así como para ser más competitivas, crear valor y sobrevivir, teniendo regulados y atendido a cada uno de los grupos de int'res del negocio" (p.538).

Estos antecedentes indica que el estudio de los Stakeholders es indispensable para las empresas en el mundo actual, debido a que su operación no será factible si no se cuenta con las apreciaciones de las partes interesadas; por tanto, sus funciones son clave para que los gerentes planifiquen la gestión de las áreas técnica, estratégica y de personal, ya que la empresa se conecta con múltiples grupos humanos, tales como: empleados, competidores, clientes, proveedores o diversas comunidades.

Un estudio analizado a nivel de Ecuador, es el denominado "Impacto de los Stakeholders en el desarrollo competitivo del sector industrial de la provincia de El Oro” presentado por, Carrión, R., (2015) quien sostiene que "el impacto competitivo que tienen los Stakeholders en las empresas está determinado en función de la diferenciación competitiva que logran las empresas" (p.81). Es decir, que representan una oportunidad para aquellas organizaciones que sean capaces 
de aprovechar estos criterios para apalancarse hacia el posicionamiento competitivo en el mercado.

Mientras que, respecto a la consideración de los Stakeholders en el diseño de plan publicitario, los autores González, L. y Mazaquiza, C., (2018) sostiene que "los empresarios ecuatorianos han visto a la publicidad como un gasto innecesario, por ende, se debe tomar en consideración el rediseño de la manera en que se hace publicidad para mejorar los resultados” (p.19). En este sentido, esta medida permitirá a las empresas poder diferenciarse dentro del sector y medir la eficiencia de las campañas publicitarias acompañados de un direccionamiento profesional y especializado con compañías consultoras para convertir estos planes en un verdadero éxito generando el posicionamiento de organizacional en la mente del consumidor.

A partir de estos antecedentes, se analiza conceptualmente cada una de las variables del estudio, de donde respecto a los Stakeholders, tal como lo explica, Garces, M., (2018) "son grupos que pueden influir en la consecución de los objetivos de una organización o verse afectados por ella” (p.31). Es decir, que toda empresa siempre tiene a su alrededor partes interesadas en su accionar, por ello, es necesario que encuentre maneras de gestionar a cada una de dichas partes para tomar decisiones que beneficien a sus actividades y le permitan lograr los objetivos planteados.

Por su parte, Alzate, J.; Orozco, J., (2017) manifiestan que "los grupos de interés son parte integral de las empresas y se deben gestionar cuidadosamente buscando la mejor forma de conectarse con sus requerimientos a fin de construir una imagen positiva” (p.106). De este modo, a criterio del autor, esto se traduce en credibilidad, confianza, reconocimiento, entre otros atributos que fortalecen la marca y la empresa.

En consecuencia, respecto al caso ecuatoriano, las organizaciones necesitan regirse bajo una planificación y gestión estratégica a fin de examinar sus grupos de interés para orientarse hacia aquellos grupos que influyen sobre la consecución de sus objetivos, tal como lo manifiesta, Torresano, M., (2012) "En Ecuador los grupos de interés ejercen presión para que las empresas definan una estrategia de atención a las presiones y fuerzas externas, así como las relaciones con los clientes, proveedores, empleados, financiadores, comunidades, sociedad, medios de comunicación, etc.” (p.30).

Esto indica que, en el campo del marketing y las estrategias de publicidad, el análisis de los Stakeholders tiene una importancia sustancial, ya que de las necesidades y expectativas de los grupos de interés debe nacer el plan publicitario que desee montar una empresa a fin de no sufrir equivocaciones que le hagan sufrir un desgaste tanto económico como de intereses o alcances pretendidos en determinado sector del mercado al que pertenece. Así pues, Parrales et. al., (2017) en su libro "Lineamiento estratégicos y comunicación digital" destacan que los lineamientos estratégicos responden al nivel tomador de decisiones y su fin es atender las necesidades sociales con una visión compartida, asegurando una reflexión estratégica que considere a la comunidad con todos sus actores y las relaciones que se deriven de esta. Sin duda los grupos de interés se convierten en esos lineamientos estratégicos para detectar errores y atender la calidad y la mejora continua en todo negocio.

Los autores, González, L. y Mazaquiza, C., (2018) explican que “el 28\% de las empresas ecuatorianas no realiza junto a consultoras o profesionales que direccionen su plan publicitario 
acorde a las necesidades y expectativas de sus Stakeholders, además ven a la publicidad como un gasto innecesario” (p.85). Por tanto, Se debe considerar la necesidad de rediseñar los métodos publicitarios para mejorar los resultados y que las empresas de esta industria se destaquen, pues lo más importante es que puedan medir la eficiencia de las campañas publicitarias y el posicionamiento de las empresas en base a planes que transformen en un verdadero éxito empresarial la operación de las mismas, posicionándose en la mente de los consumidores.

\section{DESARROLLO}

\section{Antecedentes teóricos}

El presente artículo se fundamenta en la "Teoría de los Stakeholders" que surge en 1983 de la mano de Edward Freeman como una polifonía a las tesis que sostenía Milton Friedman sobre el criterio de que "la única responsabilidad que tenía una empresa era con sus dueños" y plantea que "los trabajadores, proveedores, empresas de la competencia, clientes, administraciones públicas y la comunidad en general también son partes interesadas en la marcha de la organización" (Ramirez, H., 2020).

En efecto, el autor Ramirez, H., (2020) continúa explicando que:

La teoría de los Stakeholders proporciona pautas sobre la generación de varios tipos de valor mediante la gestión de las relaciones con las partes interesadas, pero sobre todo de un valor que trasciende el valor económico externo, por ello se cataloga como un valor intangible que representa el importe percibido desde un factor interno o externo a la organización, que resulta importante para la obtención y mantenimiento económico a corto, mediano y/o largo plazo de la empresa, con la finalidad de potenciar el poder de los empleados para mejorar el entorno laboral o formular condiciones de gestión del conocimiento que pueden reducir costos o proporcionar la mejora continua de la empresa en base a las estrategias de marketing diseñadas en la empresa (p.85).

Esta teoría sobre los Stakeholders evidencia que la importancia del análisis a las partes interesadas de una empresa es considerable, debido a que le ayuda a ajustar las metas y expectativas de todos para que sus proyectos se fundamenten en acciones que logren el éxito esperado.

Los autores, Volpentesta, J. et al., (2014) se refieren a la importancia de los Stakeholders de donde señalan:

Es necesario identificar a los Stakeholders como participantes de un proceso que pueden ser afectados y afectar las actividades de una organización, pues aquellos individuos o grupos requieren ser considerados como elementos claves durante la planificación estratégica porque son quienes se muestran interesados en el diseño de un producto o servicio que logre satisfacer sus necesidades y generar un mejor nivel de productividad (p.13).

Este criterio sostenido por los citados autores recalca la necesidad de que las empresas para poder generar valor e impacto necesitan considerar, analizar y estudiar el pensamiento de los Stakeholders como agentes que están conectados directamente con la satisfacción o insatisfacción de sus necesidades según el comportamiento de la organización y de esta manera trabajar el 
camino para cumplir con la visión de la compañía, sus objetivos y su sostenibilidad en el tiempo y el espacio.

\section{Tipos de Stakeholders}

Según, Platzy, D. (2018) indica que: Las empresas tienen al menos dos tipos de Stakeholders: los internos y los externos, los primeros son aquel grupo de personas que colaboran con la elaboración de los productos o servicios de la organización como los empleados, gerentes, colaboradores, mentores; mientras que los Stakeholders externos son aquellos que afectan directa o indirectamente porque son personas ajenas a la organización como los proveedores, gobierno, clientes o hasta la competencia (p.3).

Esto explica que siempre es necesario para el empresario identificar a qué tipo de Stakeholders va a direccionar su atención, ya que los Stakeholders primarios o internos, suelen interesarse en el funcionamiento normal de la empresa cuyo vínculo es netamente económico o de interés de crecimiento porque se trata de colaboradores, accionistas, socios, directivos, pero también existen los clientes a quienes no les interesa el factor económico sino el nivel de satisfacción que a sus necesidades que la empresa le puede ofrecer; mientras que los Stakeholders secundarios o externos no participan directamente de la empresa, pero van a permanecer interesados en los resultados de la misma como los competidores, el mercado o las personas en general.

\section{Importancia de la satisfacción de los Stakeholders}

De acuerdo con Parra, C. (2019) quien sostiene que "la satisfacción es uno de los pilares fundamentales en los que se basa la relación entre las organizaciones y los grupos de interés” (p.2).

Es decir que los Stakeholders necesitan ser complacidos de acuerdo al grado de influencia que tienen sobre la empresa, pues la valoración de los grupos de interés tienen una característica del producto o servicio que proporciona un resultado de agrado derivado del consumo, incluyendo niveles por encima o por debajo de lo esperado; la satisfacción en los grupos de interés se genera por diferentes motivos que van desde el incremento de rendimiento y la eficiencia que son generados por la innovación cómo sus deseos de satisfacción.

Por su parte, Martínez, J.; Blanco, A. y Prado, A., (2019) sostienen que "la aplicación de estrategias adecuadas generará un incremento en el nivel de satisfacción de los grupos de interés lo que implica que se debe realizar una planificación a largo plazo que sustente la legitimidad organizacional” (p.7).

Esto reafirma la necesidad de generar un tratamiento de los grupos de interés con estrategias de comunicación integral, que influyan sobre las diferencias significativas entre grupos de acuerdo a sus emociones experimentadas a fin de que se vuelvan motivadoras, traduciéndose en emociones positivas; mientras contrariamente, un Stakeholder insatisfecho puede causar perjuicios financieros para la empresa por medio de su influencia negativa o actitud que no sea de su agrado por su tiempo intereses, esto supone realizar inversiones de capital para ganar o perder dinero según las acciones y estrategias adoptadas por la empresa.

\section{Los Stakeholders como insumo de entrada}


Los autores, Masaquiza, C.; Ballesteros, L. y González, L., (2017) afirman que “debido al tabú causado por los altos precios, las pequeñas empresas generalmente no consideran el estudio de los Stakeholders en el asesoramiento publicitario por su difícil accesibilidad al presupuesto de emprendedores y gerentes" (p.7). Por su parte, Salvador, J., (2015) concuerda que "las empresas tienen el reto de identificar los grupos de interés del entorno donde se desarrollan y su impacto económico social, de riesgo y ambiental que se perciben como influencia en la adopción de mejores prácticas de gestión” (p.13).

Otro criterio es el de, García, I., (2017) quien sostiene que "desde el punto de vista publicitario de la relación entre el público objetivo y los Stakeholders hace referencia al público al cual va dirigido el plan publicitario, con el objeto que realicen las compras” (p.37).

Es decir, que se predestinan hacia un público más amplio y diverso que ha evolucionado para transformarse en un sector de interés para las empresas al momento de adquirir un plan publicitario, esto explica que la participación de los grupos de interés en la gestión de los Stakeholders en el proceso de diseño de productos publicitarios puede afectar las actividades de una empresa como elementos claves durante la planificación estratégica que esta realice.

\section{La no consideración de los grupos de interés y su repercusión en los planes publicitarios}

Empleando las palabras de Colombo, D., (2020) quien sostiene que "el hecho de que los empresarios no consideren las necesidades de sus grupos de interés ha creado un desequilibrio en las ganancias, porque están interesados en construir estrategias publicitarias empíricas en lugar de medir su impacto" (p.64).

Desde el punto de vista de Gil, A. y Paula, L., (2015) "los principales desafíos a los que se enfrentan las empresas en la búsqueda de la sostenibilidad empresarial a través de una gestión eficaz de sus grupos de interés” (P.21).

Es decir, que, para hacer frente a los retos publicitarios, las empresas están experimentando cambios profundos en las formas de mejorar la eficiencia, la eficiencia y la transparencia a través de la competitividad de la economía con nuevas formas de medir el desempeño de nuevos mecanismos de difusión e introducción de herramientas para mejorar la gestión interna de los grupos de interés.

Mientras que, Ramirez, H., (2015) indica que los Stakeholders "son los actores sociales que debido a las decisiones que toman las empresas pueden verse afacetadas tanto como negativa 0 positivamente identificando oportunidades para generar ganancias” (p.10).

En efecto, estos beneficios, buscan oportunidades para que los interesados adopten posturas a fin de analizar su nivel de interés en el proyecto y en base a eso, hacer un pronóstico de los resultados que se obtendrá y cuál será el efecto que causara en los grupos de interés.

Otro criterio es el que sostiene García, I., (2017) "la estrategia empresarial y la estrategia de marketing y publicidad brindan una guía en la elaboración del presupuesto de publicidad y objetivos organizacionales con trazabilidad" (p.223).

En efecto, las empresas ecuatorianas en algunos casos carecen de información y datos de inversión en planes publicitarios con información de los beneficios sugeridos por su grupo de interés como una herramienta imprescindible para crecer y permanecer en el mercado a largo plazo.

126 UNESUM-Ciencias. Publicación cuatrimestral. Vol. 5, Año 2021, No. 5 (Septiembre-Diciembre) 


\section{Diseño de un plan publicitario}

Según, Ramirez, H., (2015) "la relación entre las investigaciones previas y los planes publicitarios donde se considera los requerimientos de los Stakeholders son muy escazas tanto a nivel académico como empresarial” (p.16).

Esto indica que existe un bajo preámbulo de análisis y determinación de la importancia de las empresas consultoras de diseño, implementación y evaluación de planes publicitarios por parte de los empresarios según los requerimientos de los consumidores, para permanecer en su mente.

Mientras que, Masaquiza, C.; Ballesteros, L. y González, L., (2017) echan de ver el contraste investigativo argumentando que "las empresas del sector comercial a nivel mundial generan mayores ingresos y fuentes de trabajo debido al gran manejo de la imagen corporativa y estudios priorizados de agencias de consulta publicitaria” (p.16).

Es decir, que la planificación estratégica del marketing es un proceso de vital importancia para que una empresa se permita realizar una investigación de mercado y descubrir las oportunidades existentes, a fin de establecer su propia posición en ella y definirá un plan de acción para controlar su implementación, desarrollo y resultados.

Por su parte, González, M., (2015) define al diseño del plan publicitario como "el soporte económico del mensaje hacia los medios digitales e impresos que, al momento de no tener un mensaje, esta deja de existir" (p.11).

Mientras que, Cala, I, (2020) se refiere a la forma en que el plan publicitario va adquiriendo importancia en el mundo empresarial como herramienta que ayuda a comunicar a la audiencia los valores de un producto y/o servicio, de donde sostiene:

Las agencias de consultoría publicitaria y su desempeño en las entidades comerciales son el eje principal de la empresa, directa o indirectamente como su plan publicitario para empresas que tienden a confundir a los diseñadores gráficos con consultores que brindan asesoría sobre la imagen que la empresa debe proyectar en el entorno a través de materiales publicitarios impresos, y brindan asesoría a los emprendedores que usualmente ignoran el entorno externo e interno porque no tienen el impacto deseado en los consumidores (p.19).

Por tanto, la publicidad es definida como la plataforma en el cual intervienen los medios comerciales, la competitividad, las estrategias de marketing y publicidad afinando las ofertas que satisfacen las necesidades del cliente, por tanto, el plan publicitario en la actividad y el crecimiento empresarial se caracteriza por tener un bajo presupuesto al Marketing y los planes publicitarios, en especial las empresas comerciales quienes se abstienen hacer uso de herramientas comunicacionales que divulguen sus ofertas, pese a este escenario pesimista, las agencias de consulta publicitaria (ACP) sugieren que una inversión adecuada de tiempo, recursos e insumos, pues las empresas deben mantener una comunicación constante no solo a nivel de mensaje sino también visualmente, como una manifestación de la importancia del plan publicitario que puede ayudar a mantener tu imagen de marca en todos los canales. 


\section{Los Stakeholders como insumo de entrada en el plan publicitario. Casos de empresas en América Latina y Ecuador}

A nivel de América Latina se analizaron cinco empresas, cuyos casos de éxitos se detallan a continuación:

\section{Tv Globo}

La empresa Tv Globo es una organización de comunicación brasileña cuya razón de ser es ofertar espacios publicitarios para empresas, compañías y corporaciones nacionales y multinacionales, misma que ha implementado un plan publicitario en consideración a sus grupos de interés o Stakeholders que lo constituyen aquellos individuos u organizaciones que influye fuertemente en los resultados y objetivos establecidos desde los clientes hasta los accionistas, esta empresa se desenvuelve en medios convencionales, no convencionales y plataformas en la web ya que su contenido publicitario se basa en materiales audiovisuales, impresos, cable, telecomunicaciones e internet, cuyo resultado lo ha llevado a posicionarse como la cadena televisiva más influyente del país generando impacto en los accionistas, inversionistas y bancos que están conectados en la insatisfacción de su comportamiento (Orozco, J. y Ferré, C., 2015).

\section{Empresa "Diego Panesso Catering”}

Como explica, Villa, A., (2015) la empresa "Diego Panesso Catering” de nacionalidad Colombiana, dedicada a prestar servicios de soluciones integrales de alimentos y bebidas o Catering, ha diseñado su plan publicitario en base al criterio de sus Stakeholders, donde se pudo determinar que:

Respecto a la manera en que la organización se contacta con sus grupos de interés es del 27,8\% de manera telefónica, el $11,7 \%$ a través del correo electrónico y el 30,5\% de manera presencial y $70 \%$ mediante redes sociales, lo que le ha permitido definir claramente los procedimientos a seguir con los clientes y demás interesados, generando un gran impacto mostrándose como una organización seria en su trabajo con objetivos claros y organizada, ya que por medio de los Stakeholders se permite ajustar, encaminar y unificar los objetivos fundamentales de la organización con sus acciones, involucrando cada área de la compañía y generando una cultura diferente de marketing y publicidad dentro de las empresas de la región (p.163).

\section{Empresa "Bellamia"}

La empresa "Bellamia" es una organización dedicada al diseño y comercialización de lencería infantil, ubicada en la ciudad de Córdoba-Argentina, quien decidió realizar un diagnóstico de la empresa así como de su entorno y con los obtenidos los resultados procedió a diseñar y desarrollar un plan publicitario considerando el criterio de sus grupos de interés, a través de la identificación de los gustos y preferencias de los consumidores, la necesidad de posicionar la marca, identificación de los atributos distintivos de sus productos y desarrollo su estrategia de comercialización, así como de sus estrategias y tácticas (o acciones), hecho que le permitió lograr el posicionamiento como empresa líder en el mercado de ropa interior y trajes de baño para niñas, haciéndola reconocida por la variedad, originalidad de productos y diseños y su relación calidad/precio, como puntos fuertes que diferencian a Bellamia de los competidores entre un corto y mediano plazo; la forma en que esta empresa contactó a su grupo de interés fue muy diversa, utilizó medios convencionales y no convencionales de comunicación interna y externa (Apaolaza, M., 2015).

128 UNESUM-Ciencias. Publicación cuatrimestral. Vol. 5, Año 2021, No. 5 (Septiembre-Diciembre) 


\section{Empresa ALUMITEX}

La Empresa Aluminex, es una organización a dedicada a la producción y comercialización de productos en aluminio, hierro, acero inoxidable y vidrio, ubicada en Montería-Colombia.

La organización diseño un plan marketing digital con el objetivo de publicitar a través del uso de Internet y las redes sociales los productos que comercializa y los servicios que ofrece en el mercado local desde el aprovechamiento de sus ventajas competitivas ya que al realizar un análisis sobre la competencia, se determinó que las demás empresas no cuentan con procesos de marketing digital estructurados que permitieran gestionar el mercado y la relación con su público interno y externo, en efecto, desde la gerencia se lideraban acciones de acciones de marketing con alto nivel de espontaneidad y empirismo, por lo que este plan de marketing e publicitario consitituye una necesidad expresa de sus accionistas para mejorar los indicadores comerciales de la unidad de negocios, servicios especiales y enfoque relacional a bajo costo (Muñoz, C. y Cuervo, C., 2019).

\section{Empresa GEN DIGITAL}

La empresa Gen Digital, de nacionalidad peruana, logró su crecimiento desde el 2017 al 2019 gracias a una investigación cualitativa con la técnica focusgroup dirigido a personas que hayan trabajado o estén trabajando con agencias de marketing digital, donde el resultado evidenció que el problema principal era que no dedicaba tiempo para asimilar el perfil de la empresa y del cliente, optando finalmente por estrategias genéricas que le permitieran fortalecer estas debilidades e incorporar en su plan publicitario el portafolio de otras agencias también iniciantes como GlobPerú, y Carambola, a fin de que todas estas empresas cuentan con un único plan rígido de soluciones digitales que parte con una investigación preliminar sobre la empresa y termina con la creación de la página web y los perfiles en redes sociales, esta sinergia basada en el análisis de la competencia le otorgó una mayor variación en la profundidad de la investigación y el seguimiento posterior respecto a los problemas y desafíos que Gen Digital se desafío explotar (Marroquín, A., 2019).

A nivel de Ecuador se analizaron cinco empresas, cuyos casos de éxitos se detallan a continuación:

\section{Global ReportingInitiative}

Otro caso de éxito es el de la empresa fabricadora de muebles “Global ReportingInitiative” de la ciudad de Quito-Ecuador , analizado por Pacheco, V., (2015) quien indica que:

Cada Stakeholder incide sobre los resultados y el rango de riesgo y éxito de la empresa, considerando que el criterio de "los accionistas e inversionistas" incide en el 11,40\%, el sector "Gobierno" lo hace en el 29,80\%, la "Comunidad o sociedad" incide en el 29,26\%. Mientras que en la categoría de inestables se encuentran las "Personas que integran la empresa" quienes inciden con su criterio en el 68.84\%, los "Proveedores" con el 55,61\%, la "Competencia" con el $57,71 \%$ y las "Generaciones futuras" con el 43,54\% (p.58). 
Julio C. González Bennett, Jackson V. Lino Figueroa, Laura Patricia Muñiz Jaime, María Leonor Parrales Poveda

\section{Empresa SEGURLIM}

La empresa SEGURLIM, es una organización dedicada a ofrecer servicios de seguridad en la ciudad de Guayaquil-Ecuador. En el estudio se consideró la participación de 331 empresas clientes de naturaleza industrial de donde se logró determinar que el $91 \%$ necesitan que en la publicidad se indiquen los insumos de seguridad que SEGURLIM ofrece, por tanto, estos resultados obligaron a la empresa a describir en sus poster e instrumentos publicitarios los uniformes y cada uno de los insumos con que cuenta el personal como estrategia de promoción de la oferta de servicio; por otra parte se consideró a 12 empresas a las que se les consulto el nivel de satisfacción con el servicio, las cuales respondieron en un 100\% que se encuentran satisfechas con la oferta de seguridad que la organización brinda (Yánez, J., 2015).

\section{Empresa ADELCA C.A.}

Las empresas Adelca C.A. son empresas dedicadas a la fabricación de acero en el Ecuador, quienes en su plan publicitario decidieron publicar "Memorias de Sostenibilidad" a pedido de sus clientes que de manera voluntaria se lo propusieron y ellos como empresa decidieron planificar y gestionar el cambio de una manera más efectiva, así mismo, las "Memorias de Sostenibilidad” realizadas bajo las directrices de los expertos, permitieron que las empresas sean más transparentes al momento de medir y publicar sus productos, lo que les ha permitido obtener un desarrollo empresarial sostenible, ya que se genera confianza tanto a nivel interno como externo con todos sus Stakeholders, minimizando las presiones generadas por los mismos, además de constituirse como una herramienta estratégica para la comunicación de las empresas (Cobo, J., 2016).

\section{Supermercados TÍA}

En un estudio publicado por, Sáenz, G., (2018) analiza la relación con los Stakeholders mediante la red social Facebook de los Supermercados TíA, donde indica que, 192 clientes de guayaquil y 192 clientes de Quito (Ecuador), permitieron conocer que esta empresa trabaja arduamente para ser reconocida y respetada dentro del mercado ecuatoriano brindando productos de calidad a precios asequibles, lo que genera como consecuencia la construcción de una buena imagen tanto de sus clientes internos y externos (p.80).

\section{La Fortaleza S.A.}

La empresa florícola "La Fortaleza S.A.” se encuentra ubicada en Imbabura-Ecuador, misma que tras realizar un análisis sobre sus clientes logró determinar que no cuenta con una muy buena aceptación en el mercado floricultor, no posee una imagen que identifique sus productos, no cuenta con un análisis competitivo, ofrece limitados productos al mercado y pocos clientes conocen de la existencia de sus productos en el mercado, hecho que la llevó a identificar que le falta un estudio de marketing y estrategias de publicidad, por lo que ha decidido diseñar un plan publicitario en base a estos requerimientos identificados para mejorar la comercialización de sus productos (Ontaneda, G., 2017).

\section{Materiales y métodos}

En el presente estudio se analizan caso de éxitos del estudio de los Stakeholders como insumo de entrada para el diseño de un plan publicitario en cinco empresas latinoamericanas y cinco empresas ecuatorianas, para tal efecto, se utilizó una investigación de tipo descriptiva, apoyada

130 UNESUM-Ciencias. Publicación cuatrimestral. Vol. 5, Año 2021, No. 5 (Septiembre-Diciembre) 
en los métodos deductivo e inductivo. Se emplea el método deductivo porque se busca obtener datos sobre los hechos concretos que se relacionan al estudio de los Stakeholders; se emplea el método de la inducción porque se parte de un tema específico que apunta a la generalidad con el fin de establecer conclusiones verdaderas y oportunas sobre la importancia que tienen los grupos de interés en el diseño de un plan publicitario, de modo que los investigadores han logrado comparar criterios planteados a través de los resultados y conclusiones de investigación consultadas en diferentes sitios de la web.

El camino considerado para llegar a establecer los resultados de este estudio está fundamentado en la revisión de una considerable literatura y compilaciones bibliográficas de diversos recursos de consultoría en línea, tales como revistas científicas regionales indexadas y repositorios digitales con el propósito de organizar los distintos esquemas y criterios sostenidos por los autores que han tratado las variables de estudio con anterioridad, generando espacio para la inferencia y punto de vista del equipo investigador a fin de encontrar resultados novedosos sobre este tema para analizar los Stakeholders como insumo de entrada en el diseño de un plan publicitario.

\section{Discusión}

Pese a que con el gran avance a nivel empresarial, económico y social que se vive en el mundo, existen empresas que consideran que el diseño de un plan publicitario en el que se analice a los Stakeholders es un gasto innecesario, existen también empresas que se consideran como "casos de éxito" que se han arriesgado a tratar el estudio de los Stakeholders con la importancia que este sector requiere y han visto reflejado de manera positiva la capacidad de desarrollo y sostenibilidad a largo plazo, tal es el caso de las diez empresas objeto de estudio, ellas son: Tv Globo (Brasil), "Diego Panesso Catering” (Colombia), "Bellamia” (Argentina), ALUMITEX (Colombia) y GEN DIGITAL (Perú), así como las empresas Global ReportingInitiative, SEGURLIM, ADELCA C.A., Supermercados TÍA y La Fortaleza S.A. de Ecuador que han logrado su éxito empresarial en un corto y mediano plazo.

Estos resultados son acordes a lo que sostienen, Volpentesta, J. et al., (2014) quienes consideran que es importante para todas las empresas identificar a los Stakeholders y establecer mecanismos de participación como elementos claves para fortalecer la planificación del contenido publicitario a fin de que apunte hacia las necesidades del cliente y mejore la productividad empresarial, como lo indica la "Teoría de los Stakeholders" planteada por Edward Freeman quien sostiene que la responsabilidad de la empresa no solo es con sus dueños, sino también con los trabajadores, proveedores, la competencia, clientes, administraciones públicas y sociedad en general.

Existen diferentes formas de contactar a los grupos e interés por parte de las empresas, en este sentido se usan medios convencionales como prensa, radio, televisión, revistas; medios no convencionales como: eventos de ferias, anuarios, guías y boletines, etc.; y las plataformas digitales como: redes sociales y buscadores web que conducen a páginas de la empresa o blogs organizacionales, de los casos analizados, el $10 \%$ utiliza medios convencionales, el $20 \%$ lo hace a través de medios no convencionales y el $70 \%$ mediante plataformas digitales, lo que permite abaratar costos de mercadeo. 
Estos resultados guardan relación con lo que indican Gil, A. y Paula, L., (2015) quienes afirman que los desafíos empresariales del análisis de los Stakeholders como insumo de entrada publicitaria es la búsqueda de la sostenibilidad empresarial a través de una gestión eficaz de la información que los grupos de interés aporten a la organización y el coste de los medios o canales de comunicación que tengan las empresas con sus partes interesadas.

Los Stakeholders son un grupo esencial para el funcionamiento de las empresas nacionales y extranjeras, ya que estos grupos constituyen una burbuja en las decisiones que afectan directa o indirectamente sobre los resultados y objetivos organizacionales de acuerdo a las actividades publicitarias y de diseño de productos o servicios que éstas incorporen a su gestión, en efecto de $100 \%$ de los casos citados ha conseguido resultados positivos tras considerar como factor esencial a los Stakeholders para el desarrollo empresarial y posicionamiento en el mercado al que pertenecen.

Esto es acorde al criterio de González, M., (2015) quien considera que las partes interesadas son el interés público de la empresa que le permite operar plenamente, así como establecer actividades, objetivos y tomar decisiones en torno al pensamiento emitido por los empleados, proveedores, clientes, gobiernos, competencia, etc.

\section{CONCLUSIONES}

Según el análisis de la revisión bibliográfica se establecen las siguientes conclusiones:

Las empresas ecuatorianas necesitan asumir un mayor compromiso con el crecimiento organizacional, pero necesitan considerar las expectativas de los Stakeholders a fin de definir la función de su percepción de valor que aporte al desarrollo estratégico de la empresa acorde a los requerimientos potenciales de su grupo de interés.

La relación que deben tener las empresas con los Stakeholders necesita un nivel de heterogeneidad que fortalezca las capacidades de diferenciación y sostenibilidad a través del capital humano, la estructura organizativa y el plan publicitario que se pueda implementar según las necesidades de las partes interesadas que se relacionan directamente con el valor de la empresa.

Los Stakeholders constituyen el insumo de entrada para la planificación publicitaria que la empresa desee ejecutar ya que el posicionamiento competitivo en el mercado depende directamente de los involucrados en la vida de la organización para que se puedan generar los beneficios de manera proactiva hacia los objetivos planteados, en base a métodos para relacionar las prácticas de responsabilidad social y el grado de institucionalización formal de la gestión asociada a ellos.

\section{REFERENCIAS BIBLIOGRÁFICAS}

Alzate, J.; Orozco, J. (2017). Stakeholders, actores estratégicos en la construcción de marca. Universidad de Medellín. Obtenido de http://www.scielo.org.co/pdf/angr/v16n32/1692-2522-angr-16-32-95.pdf

Apaolaza, M. (2015). Plan de Marketing y Estrategia de posicionamiento para un emprendimiento de lencería infantil: Bellamia. Universidad Nacional de Córdoba. Obtenido de

132 UNESUM-Ciencias. Publicación cuatrimestral. Vol. 5, Año 2021, No. 5 (Septiembre-Diciembre) 
https://rdu.unc.edu.ar/bitstream/handle/11086/4064/Apaolaza\%2C\%20Maria\%20Fernanda.\%20Plan\%20de \%20Marketing\%20y\%20Estrategia\%20de....pdf?sequence=1\&isAllowed=y

Cala, I. (2020). Importancia del diseño gráfico y la publicidad. Factoría Creativa. Obtenido de https://www.factoriacreativabarcelona.es/blog/la-importancia-del-diseno-grafico-publicidad/

Carrión, R. (2015). Impacto de los Stakeholders en el desarrollo competitivo del sector industrial de la provincia de El Oro. Universidad del Azuay. Obtenido de http://dspace.uazuay.edu.ec/bitstream/datos/3729/1/10398.pdf

Cobo, J. (2016). Estudio de nuevo modelo de Gestión de RSC 2.0 para la obtención del certificado S2M. Caso de estudio Acerias del Ecuador ADELCA C.A. periodo 2008-2014. Pontificia Unoversidad Católica del Ecuador.

Obtenido http://repositorio.puce.edu.ec/bitstream/handle/22000/11529/ESTUDIO\%20DEL\%20NUEVO\%20MODEL O\%20DE\%20GESTI\%C3\%93N\%20DE\%20R.S.C.\%202.0\%20PARA\%20LA\%20OBTENCI\%C3\%93N\% 20DEL\%20CERTIFICADO\%20S2M.\%20CASO\%20DE\%20.pdf?sequence=1\&isAllowed=y

Colombo, D. (2020). Así debes luchar contra la falta de profesionalismo en tu equipo de trabajo. Obtenido de https://www.entrepreneur.com/article/345464

Garces, M. (2018, Julio). Marketing y Gestión de Negocios. Obtenido de http://repositorio.uta.edu.ec/jspui/handle/123456789/28440

García, I. (2017). Presupuesto de marketing y evaluación de beneficios generados por el producto. Obtenido de https://repositorio.espe.edu.ec/bitstream/21000/1154/6/T-ESPE-021513-6.pdf

Gil, A. y Paula, L. (2015). La gestion de los grupos: una reflexion sobre los desafios a los que se enfrentan las empresas en la busqueda de la sostenibilidad empresarial. Revista de métodos cuantitativos para la economía y la empresa, 71-90. Obtenido de https://dialnet.unirioja.es/servlet/articulo?codigo=3657902

González, L. y Mazaquiza, C. (2018). Análisis de los stakeholders como insumo de entrada en el diseño de un plan publicitario. Universidad Técnica Ambato. Obtenido de https://repositorio.uta.edu.ec/jspui/handle/123456789/28440

González, M. (2015). Análisis del uso de los conceptos de público, stakeholder y constituent en el marco teórico de las relaciones públicas. Obtenido de https://www.researchgate.net/publication/28214523_Analisis_del_uso_de_los_conceptos_de_publico_stake holder_y_constituent_en_el_marco_teorico_de_las_relaciones_publicas

Marroquín, A. (2019). Plan de marketing para la empresa GEN DIGITAL Perú - 2019. Universidad San Ignacio de Loyola. Obtenido de http://repositorio.usil.edu.pe/bitstream/USIL/9257/1/2019_Marroquin-Vidal.pdf

Martínez, J.; Blanco, A. y Prado, A. (2019). Un análisis de la satisfacción de los stakeholders y la legitimidad: El modelo de negocio de la institución universitaria. Revista Espacios. Obtenido de http://www.revistaespacios.com/a19v40n16/a19v40n16p27.pdf

Masaquiza, C., Ballesteros, L., \&Gonzalez, L. (2019).

Masaquiza, C.; Ballesteros, L. y González, L. (2017). Análisis de los Stakeholders como insumo de entrada en el diseño de un plan publicitario. Universidad, Ciencia y Tecnología. Obtenido de http://www.uct.unexpo.edu.ve/index.php/uct/article/download/823/669

Moreno, R. . (2015). Los Stakeholders y la Responsabilidad Social Corporativa. Revista Cumbria FSC. Obtenido de https://www.iimv.org/iimv-wp-1-0/resources/uploads/2015/03/Capitulo-13-.pdf

Muñoz, C. y Cuervo, C. (2019). Propuesta de un plan de marketing Digital para la empresa ALUMITEX de la ciudad de Monteria. Universidad Coperativa de Colombia. Obtenido de https://repository.ucc.edu.co/bitstream/20.500.12494/7180/2/2019_propuesta_plan_marketing.pdf 
Julio C. González Bennett, Jackson V. Lino Figueroa, Laura Patricia Muñiz Jaime, María Leonor Parrales Poveda

Ontaneda, G. (2017). Elaboración de plapublitario de la empresa florícola "La fortaleza S.A. Leoforta" de Imbabura. Universidad de las Fuerzas Armadas. Obtenido de https://repositorio.espe.edu.ec/bitstream/21000/12838/1/M-ESPE-053730.pdf

Orozco, J. y Ferré, C. (2015). Los Stakeholders de las empresas de comunicación en el ámbito de la responsabilidad social corporativa. Revista Folios. Universidad de Antioquia.

Pacheco, V. (2015). Diagnóstico de sostenibilidad para valorar la gestión de responsabilidad social (RSE) en las PYMEs del distrito metropolitano de Quito. Universidad Católica del Ecuador. Obtenido de http://repositorio.puce.edu.ec/bitstream/handle/22000/12261/TESIS\%20VICTOR\%20PACHECO.pdf?sequ ence $=1 \&$ isAllowed $=\mathrm{y}$

Parra, Camilo. (2019). Aplicación de la gestión de los Stakeholders en proyectos de onvestigación en Colombia. Cartagena - Colombia: Universidad Tecnológica de Bolívar. Obtenido de https://biblioteca.utb.edu.co/notas/tesis/0073238.pdf

Parrales, M.L.; Gracia, E.A.; Miller, J.H.; Ponce, O.S. (2017). Lineamientos estratégicos y comunicación digital. Mawil Publicaciones de Ecuador.

Platzy. Demian. (2018). Qué son stakeholders y sus tipos. Obtenido de https://platzi.com/blog/stakeholders-y-sustipos/

Ramirez, H. (2015). Propuesta de un Informe de Capital Intelectual para las instituciones de educación superior españolas. Estudios de Economía Aplicada. Obtenido de https://www.redalyc.org/pdf/301/30128236014.pdf

Ramirez, H. (2020). Stakeholders ¿Cómo impactan a una empresa y cómo gestionarlos? Obtenido de https://protecciondatos-lopd.com/empresas/stakeholders/

Sáenz, G. (2018). Dimensionalidad de la Responsabilidad Social Empresarial percibida y sus efectos sobre la Imagen y Reputación de la marca: Estudio de Caso Supermercados TÍA. Universidad Cat'lica de Guayaquil. Obtenido de http://repositorio.ucsg.edu.ec/bitstream/3317/10836/1/T-UCSG-POS-MAE-170.pdf

Salvador, J. (2015). Importancia de los grupos de interés en la adopción de prácticas de gestión ambiental de las empresas de exploración y explotación de hidrocarburos en el Perú. Universidad Ramon Llul. Obtenido de https://www.tdx.cat/bitstream/handle/10803/284803/00_2014_Julio\%20SALVADOR\%20ESADE_ESAN\% 20PhdThesis.pdf?sequence $=1 \&$ isAllowed $=\mathrm{y}$

Torresano, M. (2012). Estudio de responsabilidad social de empresas del Ecuador. AVINA. Obtenido de https://docplayer.es/71098658-Estudio-de-responsabilidad-social-de-empresas-del-ecuador.html

Villa, A. (2015). Diseño de un plan estratégico de marketing para la empresa Diego Panesso Catering. Universidad Tecnológica de Pereira. Obtenido de http://recursosbiblioteca.utp.edu.co/tesisd/textoyanexos/658812V712.pdf

Volpentesta, J. et all. (2014). Identificación del impacto de la gestión de los stakeholders en las estructuras de las empresas que desarrollan estrategias de responsabilidad social empresarial. Bogotá - Colombia: Universidad \& Empresa, vol. 16, núm. 26. Obtenido de https://www.redalyc.org/pdf/1872/187232713003.pdf

Yánez, J. (2015). Plan de Marketing para posicionar la empresa SEGURLIM en el sector de la seguridad de la ciudad de Guayaquil. Universidad Politécnica Salesiana del Ecuador. Obtenido de https://dspace.ups.edu.ec/bitstream/123456789/10011/1/UPS-GT001012.pdf 